\title{
Modelling the distribution of $\mathrm{BaTiO}_{3}$ nanoparticles in a $\mathrm{P}_{\left(\mathrm{VDF}_{70}-\mathrm{TrFE}_{30}\right)}$ polymer matrix for permittivity calculation
}

\author{
Jonas Hafnera, Jürgen Schrattenholzer, Marco Teuschel, Michael Schneider, \\ Daniel Platz and Ulrich Schmid \\ Institute of Sensor and Actuator Systems, TU Wien, Gusshausstrasse 27-29, 1040 Vienna, Austria
}

\begin{abstract}
Nanocomposites are an exciting field for both research and application related activities as they promise to push the existing limits of many standard materials. For instance, the admixture of inorganic nanoparticles into polymers may lead to materials with novel dielectric properties. However, nanocomposites are highly complex material systems, making the prediction of basic material parameters, such as the permittivity, challenging. In this study, we present a novel method that considers explicitly the statistical distribution of the nanoparticles in the organic matrix, which allows to calculate the permittivity as a function of the nanoparticle volume fraction. We apply this method for the study of spherical barium titanate particles in a poly(vinylidene fluoride ${ }_{70}$-trifluoroethylene 30 ) polymer matrix and show that a transition from randomly distributed nanocomposites to colloidal crystals occurs, which has a tremendous impact on the permittivity of the composite.
\end{abstract}

Keywords: Polymer-matrix composites; electrical properties; computational modelling

(C) 2019. This is the peer reviewed version of the following article: Hafner, J., Schrattenholzer, J., Teuschel, M., Schneider, M., Platz, D. \& Schmid, U. Modelling the distribution of $\mathrm{BaTiO}_{3}$ nanoparticles in a $\mathrm{P}\left(\mathrm{VDF}_{70}-\mathrm{TrFE}_{30}\right)$ polymer matrix for permittivity calculation. Polymer 180, 121682 (2019), which has been published in final form at https://doi.org/10.1016/j.polymer.2019.121682. This manuscript version is made available under the CC-BY-NCND 4.0 license http://creativecommons.org/licenses/by-nc-nd/4.0/.

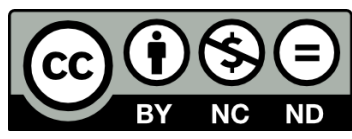

${ }^{\mathrm{a}}$ E-mail: jonas.hafner@tuwien.ac.at 


\section{Introduction}

Polymers and ceramics are among the most important classes of materials for electronic applications. Ceramics like barium titanate $\left(\mathrm{BaTiO}_{3}\right)$ or lead zirconate titanate (PZT) are nowadays the basis for the realization of countless electronic devices, such as capacitors ${ }^{1-3}$, but also sensors ${ }^{4-6}$ and actuators ${ }^{7,8}$. Compared to other materials, these ceramics are well-known for their remarkable high permittivity ${ }^{9,10}$, but suffer from their low breakdown strength. ${ }^{11,12}$ In contrast, polymers offer a substantially higher dielectric strength, but their permittivity is comparably low. ${ }^{13}$ Hence, combining these two materials can result in novel multiphase materials exhibiting both a high breakdown strength and a high permittivity. ${ }^{14-17}$ The inclusion of ceramic nanoparticles with a size of about $100 \mathrm{~nm}$ into a polymer matrix opens up a new route for the realization of dielectric capacitors with higher energy densities compared to the competing electrochemical supercapacitors. With regard to the growing interest in flexible and soft electronics, polymer-based nanocomposites offer many possibilities in this emerging field of application. ${ }^{18,19}$

So far, many polymer-based nanocomposites have been studied in terms of their dielectric and mechanical properties. ${ }^{14-17,20-22}$ In particular, the modelling of these complex material systems in dependence of the volume fraction of the included nanoparticles was of utmost importance. These investigations led to a large variety of theoretical approaches, such as those of Maxwell-Garnett ${ }^{20}$, Yamada $^{23}$, Lichtenecker $^{20,24}$ or the self-consistent effective medium theory $\left(\right.$ SC-EMT) ${ }^{14}$, to predict the permittivity of the nanocomposites. However, none of these studies explicitly consider the statistical distribution of the particles inside the composite. Although included in various approximations, the nanoparticle distribution is crucial for the prediction of the overall permittivity, especially at enhanced particle density values. For a volume fraction between 40 and $50 \%$ of spherical nanoparticles Kim et al. showed that there is a threshold value at which the permittivity of the composite suddenly starts to decrease. ${ }^{14}$ Under these conditions, the nanoparticles no longer find any free space in the spin-coated nanocomposite film and start to accumulate close to the free surface of the film as a porous layer, which 
in turn reduces the permittivity and the breakdown strength. The decrease of the permittivity was included in their model by the implementation of air voids in the nanocomposite films. However, it was not investigated which permittivity can achieve in the nanocomposites in this regime, although it is possible to fabricate nanocomposite films with such high volume fractions of nanoparticles even without a porous layer. This already indicates that with increasing volume fraction, the initially random distribution of the nanoparticles must be transformed into a symmetrical arrangement. This specific particle distribution can not be achieved by a straightforward spin-coating process - the crucial formation of colloidal crystals of nanoparticles in the polymer matrix can only be achieved by introducing a more complex fabrication process, as reported in Refs. 25 and 26.

In this study, we present a new method to calculate the permittivity of the nanocomposite films based on the statistical distribution of particles in the polymer matrix. We determine the distributions of spherical nanoparticles of $\mathrm{BaTiO}_{3}$ in a polymer matrix of poly(vinylidene 70 -trifluoroethylene 30 ) $\left(\mathrm{P}\left(\mathrm{VDF}_{70}-\mathrm{TrFE}_{30}\right)\right)$ for different volume fractions and compare the theoretical results with experimental data. We calculate the permittivity for selected colloidal crystal formations in the polymer, thus demonstrating the potential of these nanocomposite films as high permittivity material. 


\section{The distribution of nanoparticles in nanocomposites and the calculation of their permittivity}

The statistical distribution of the nanoparticles inside of a polymer matrix is crucial for the permittivity calculation. Figure 1 (a) shows schematically the procedure for the uniform distribution of the particles in the matrix. Starting from a given volume fraction

$$
v_{f}=\frac{n_{p} \cdot V_{p}}{V_{t o t}}
$$

$n_{p}$ spherical nanoparticles with the same diameter $d_{p}=r_{p} / 2$ and volume $V_{p}=\frac{4}{3} \pi r_{p}^{3}$ are distributed in a polymer film with a total volume of $V_{t o t}=l_{x} \cdot l_{y} \cdot l_{z}$. For this purpose, each particle (i.e. sphere center) is randomly assigned a position $\left(x_{i}, y_{i}, z_{i}\right)$ inside the polymer. Once a particle was placed, it can not change its position anymore. During the distribution of the spheres, a minimum distance is kept between each particle, so that they can be in contact, but no overlap occurs. At the outer borders of the composite film, particles are allowed to protrude out of the surface, as long as their center is still inside of the composite film. At the top and lower film surfaces, where the electrodes are deposited to form a capacitor, the particles are not allowed to protrude out of the surface. From the distribution of the nanoparticles, the geometrical expansion (thickness)

$$
t_{i}^{p}(x, y)=2 \cdot \sqrt{r_{p}^{2}-\left(x-x_{i}\right)^{2}-\left(y-y_{i}\right)^{2}}
$$

of each particle $i$ in $z$-direction is projected onto the $x y$-plane. The superposition of each particle thickness function $t_{i}^{p}(x, y)$ gives

$$
t(x, y)=\sum_{i} t_{i}^{p}(x, y)
$$

which indicates the amount of particle material in $z$-direction at position $(x, y)$ of the nanocomposite film. Figure 1 (b) shows the total thickness function $t(x, y)$ for a volume fraction of $v_{f}=5 \%$ of spherical nanoparticles $\left(d_{p}=120 \mathrm{~nm}\right)$ distributed inside a polymer matrix (i.e. $l_{x}=3 \mu \mathrm{m}, l_{y}=2 \mu \mathrm{m}, l_{z}=1 \mu \mathrm{m}$ ). 


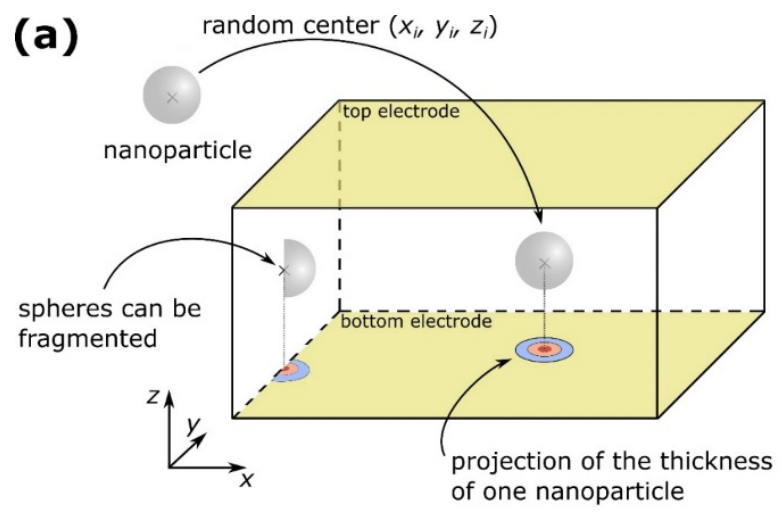

(c)

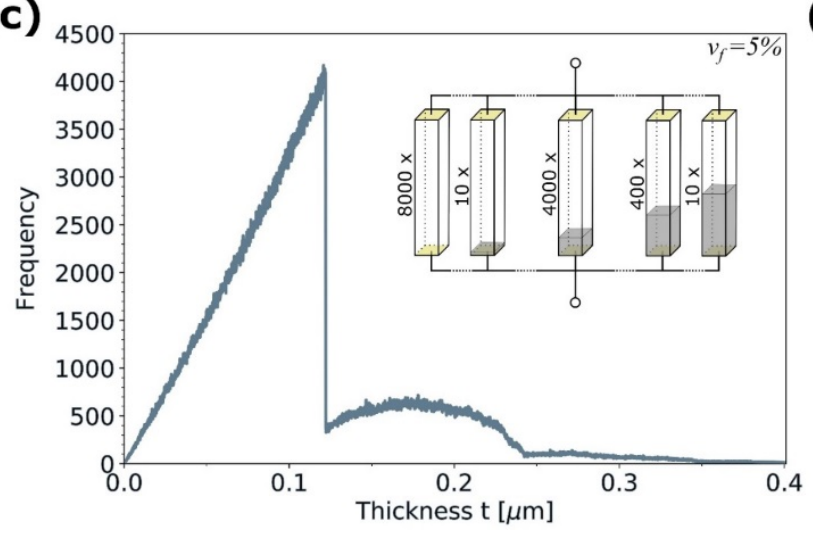

(b)

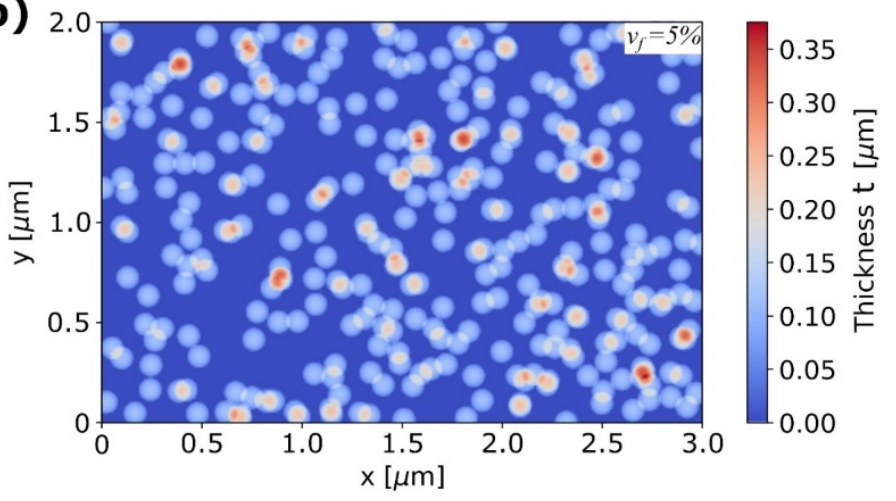

(d)

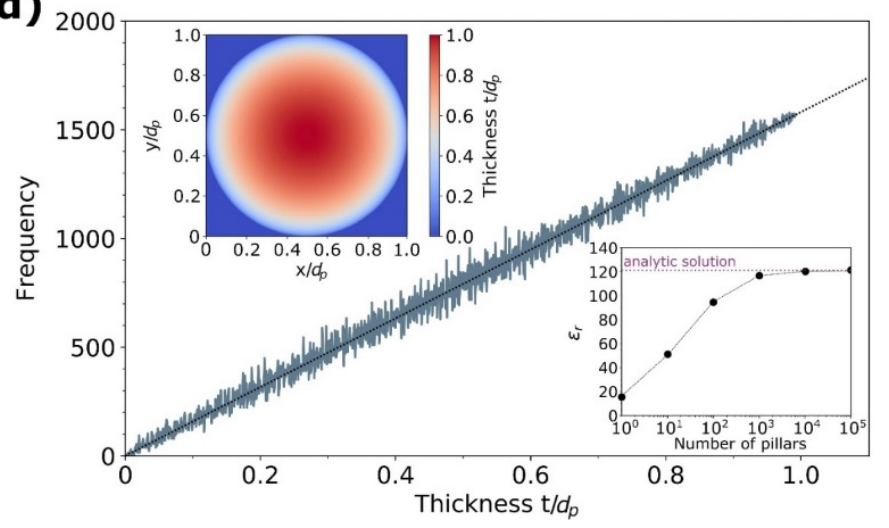

Figure 1. (a) Illustration of the algorithm for the random distribution of the nanoparticles inside of a polymer matrix. (b) The calculated total thickness function $t(x, y)$ for a distribution with a volume fraction $v_{f}=5 \%$. (c) The distribution $n\left(t_{i}\right)$ for the total thickness function $t(x, y)$ from (b). The inset illustrates the pillars (i.e. capacitors) with different amount of particle material, which are connected in parallel to each other. (d) The distribution $n\left(t_{i}\right)$ of $t(x, y)$ of one nanoparticle $\left(d_{p}=120 \mathrm{~nm}, \varepsilon_{r}^{p}=6000\right)$ in a polymer matrix $\left(\varepsilon_{r}^{m}=15\right)$.

For the calculation of the permittivity, the capacitor consisting of the nanocomposite and the bottom- and top-electrode is divided in $z$-direction into a pillar-type structure. Each of these pillars represents a capacitive element, which are connected in parallel. Every pillar $i$ has a certain thickness $t_{i}$ of the particle material with a permittivity $\varepsilon_{r}^{p}$ and a thickness $l_{z}-t_{i}$ of the polymer matrix with the permittivity $\varepsilon_{r}^{m}$. The capacitance of each pillar can be divided into two sub-elements connected in series, whereas $C_{m}$ and $C_{p}$ represent the individual capacitance values of the polymer matrix and the material of the particle, respectively. Together with the parallel connection of all pillars, the total capacitance of the 
nanocomposite film is

$$
C_{t o t}=\sum_{i} n\left(t_{i}\right) \cdot \frac{C_{p}\left(t_{i}\right) \cdot C_{m}\left(t_{i}\right)}{C_{p}\left(t_{i}\right)+C_{m}\left(t_{i}\right)} .
$$

The function $n\left(t_{i}\right)$ indicates the number of pillars with the thickness $t_{i}$ of the particle material. Figure 1 (c) shows the distribution $n\left(t_{i}\right)$ of the total thickness function $t(x, y)$ from Figure $1(\mathrm{~b})$. Here, the pillars with no particle inside have been omitted for the purpose of clarity. When knowing the distribution $n\left(t_{i}\right)$, the permittivity of the composite can be calculated from equation (4) by

$$
\varepsilon_{r}=l_{z} \cdot \sum_{i} \frac{n\left(t_{i}\right)}{n_{t o t}} \cdot \frac{\varepsilon_{r}^{p} \cdot \varepsilon_{r}^{m}}{\varepsilon_{r}^{p} \cdot\left(l_{z}-t_{i}\right)+\varepsilon_{r}^{m} \cdot t_{i}},
$$

where $n_{\text {tot }}$ is the total number of pillars. In the limit

$$
\lim _{i \rightarrow \infty} \frac{n\left(t_{i}\right)}{n_{\text {tot }}}=p(t)
$$

the discrete distribution function $n\left(t_{i}\right)$ becomes a continuous density function $p(t)$. In this case, the equation (5) simplifies to

$$
\varepsilon_{r}=l_{z} \cdot \int_{0}^{l_{z}} p(t) \cdot \frac{\varepsilon_{r}^{p} \cdot \varepsilon_{r}^{m}}{\varepsilon_{r}^{p} \cdot\left(l_{z}-t\right)+\varepsilon_{r}^{m} \cdot t} d t .
$$

Regardless of the geometry and position of the nanoparticles in the composite, it is possible to theoretically predict from the simulation of the particle distribution inside of the polymer matrix the overall permittivity of the dielectric nanocomposite.

To evaluate our approach, we determined the density function as well as the permittivity of a single spherical nanoparticle with a diameter $d_{p}=120 \mathrm{~nm}$ and a permittivity $\varepsilon_{r}^{p}=6000$ inside of a polymer matrix with the permittivity $\varepsilon_{r}^{m}=15$. The results are presented in Figure 1 (d). It turns out that the distribution $n\left(t_{i}\right)$ of the thickness function of a single nanoparticle is linear, which corresponds to the analytic behaviour $p(t) \sim t$ of a sphere. In addition, with an increasing number of pillars, the permittivity converges to the analytic value of $\varepsilon_{r} \approx 125$ (see inset of Figure $1(\mathrm{~d})$ ). 
The presented model provides a straightforward way to estimate the permittivity of complex polymermatrix composites compared to numerical methods such as the finite element method (FEM), molecular dynamics (MD) or coarse-grained (CD) simulations. FEM, MD or CG simulations can usually compute only small volumes of complex composite structures, i.e. the number of embedded particles is limited. While the approach presented here is able to calculate much larger volumes, which makes it possible to predict the impact of a wide variety of particle distributions inside of the polymer matrix. Even effects such as agglomeration can be studied over a large volume of the composite, requesting, however, an adaptation of the particle distribution by the density function $p(t)$. In addition, the distribution of the particles size can be taken into account via the density function $p(t)$. In this paper, however, it is assumed for the sake of simplicity that particles of the same size are uniformly distributed in the polymer matrix. Effects like agglomeration or the distribution of the particle size are neglected. Nonetheless, such effects can be included in the model and already other papers showed that agglomeration has a huge impact on the properties of polymer-matrix composites. ${ }^{27}$ The limitation of the model is that the electric field is assumed rectilinear throughout the composite, i.e. stray fields caused by the particles and their impact on adjacent particles are not considered. Although an interphase can be modelled between the particles and the polymer matrix by introducing an additional third phase, which envelops the particles, any kind of dielectric interaction between the particles is not readily integrated into the model. Nevertheless, the permittivity values of the fabricated polymer-matrix composites are in good agreement with those predicted by the simplified model. This model is a useful tool to estimate the permittivity over a large volume of complex composite structures, which allows analysing a wide variety of particle distributions inside of a polymer matrix with less computational effort. 
(a)

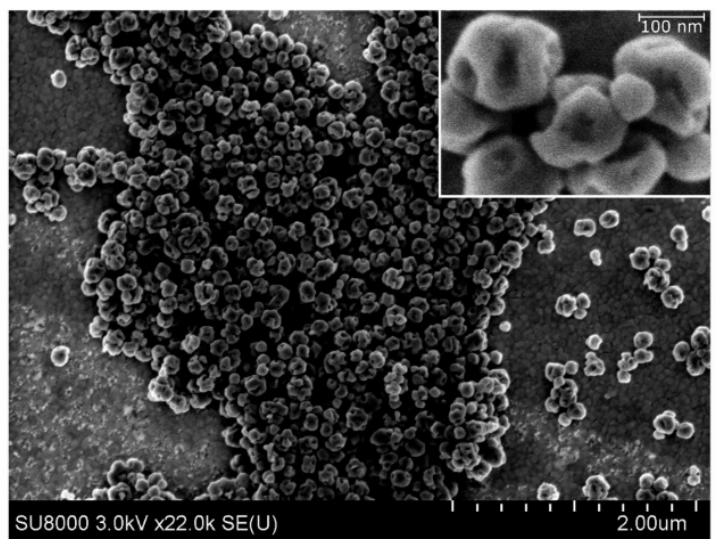

(b)

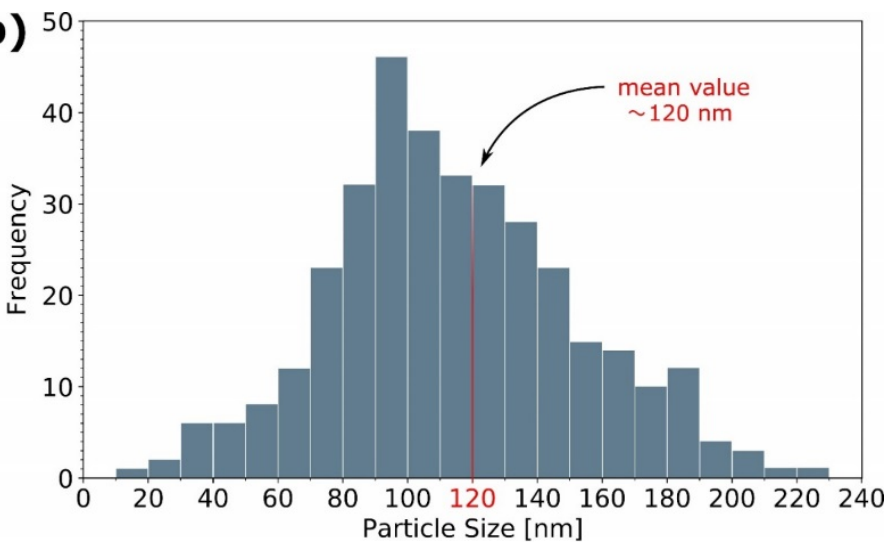

Figure 2. (a) $\mathrm{SEM}$ image of $\mathrm{BaTiO}_{3}$ nanoparticles. The inset shows an enlarged view demonstrating a poor spherical particle shape. (b) Particle size statistics based on an evaluation of 350 nanoparticles: mean particle size value is about $120 \mathrm{~nm}$.

\section{Fabricated nanocomposite films}

\section{1 $\mathrm{BaTiO}_{3}$ nanoparticle analysis}

We prepared nanocomposite films with $\mathrm{BaTiO}_{3}$ nanoparticles in a polymer matrix of $\mathrm{P}\left(\mathrm{VDF}_{70}-\mathrm{TrFE}_{30}\right)$. The spherical $\mathrm{BaTiO}_{3}$ nanoparticles are purchased from Nanografi. We analyzed the size and the geometry of the nanoparticles with a scanning electron microscope (SEM). Figure 2 (a) shows an SEM image of the nanoparticles. In addition, the inset shows that a spherical geometry is only a roughly approximation compared to the real shape of the particles. The distribution of the nanoparticle size is presented in Figure 2 (b). The diameters of the particles range from $10 \mathrm{~nm}$ to $230 \mathrm{~nm}$ with a mean value of about $120 \mathrm{~nm}$. For further considerations, this latter value will be taken as representative particle diameter. The polymer $\mathrm{P}\left(\mathrm{VDF}_{70}-\mathrm{TrFE}_{30}\right)$, which serves as matrix for the nanoparticles, was purchased in powder form from Piezotech/Arkema. This powder was dissolved at a weight ratio of $4 \%$ in the solvent methyl ethyl ketone (MEK). 


\subsection{Fabrication of $\mathrm{BaTiO}_{3} / \mathrm{P}_{\left(\mathrm{VDF}_{70}-\mathrm{TrFE}_{30}\right) \text { nanocomposites }}$}

For the fabrication of the nanocomposite films, we mixed different volume fractions of particles into the polymer solution. The added mass

$$
m_{p}=\frac{m_{m} \cdot v_{f}}{1-v_{f}} \cdot \frac{\rho_{p}}{\rho_{m}}
$$

of the nanoparticles for different volume fractions $v_{f}$ was measured $\left(m_{m}=\right.$ mass of the polymer inside of the solution, $\rho_{m}=$ density of the $\mathrm{P}\left(\mathrm{VDF}_{70}-\mathrm{TrFE}_{30}\right)$ polymer, $\rho_{p}=$ density of the $\mathrm{BaTiO}_{3}$ nanoparticles $)$. The density of the $\mathrm{BaTiO}_{3}$ nanoparticles $\rho_{p}=5.90 \mathrm{~g} / \mathrm{cm}^{3}$ is specified by the manufacturer. The density of the $\mathrm{P}\left(\mathrm{VDF}_{70}-\mathrm{TrFE}_{30}\right)$ polymer $\rho_{m}=1.82 \pm 0.05 \mathrm{~g} / \mathrm{cm}^{3}$ was calculated by measuring the mass and the volume of the spin-coated polymer films. The particles were homogenously distributed in the polymer solution for about $1 \mathrm{~h}$ using a sonicator (UP400S from Hielscher). An external water cooling system was used to prevent the solvent MEK from evaporation. Subsequently, the solution with the polymer and the nanoparticles was spin-coated onto a 4" (100) silicon (Si) wafer and the solvent MEK was carefully evaporated at $80{ }^{\circ} \mathrm{C}$ in air. This is followed by an annealing process at $130{ }^{\circ} \mathrm{C}$ for $2 \mathrm{~h}$ in a vacuum furnace to enhance the degree of crystallinity. We obtained nanocomposite films with a thickness between 0.5 and $1 \mu \mathrm{m}$ depending on the volume fraction $v_{f}$ of the admixed nanoparticles.

\subsection{Dielectric characterization of the nanocomposites}

To measure the permittivity of the nanocomposite films, we fabricated capacitors with different surface areas ranging from 1 to $50 \mathrm{~mm}^{2}$ by applying standard micromachining technologies. For this purpose, we used a 4" (100) Si wafer coated with $150 \mathrm{~nm}$ LPCVD silicon dioxide as substrate. The bottom electrodes were formed by a conventional lift-off process. $50 \mathrm{~nm}$ of titanium (Ti) serving as an adhesion promoter 
and a $200 \mathrm{~nm}$ thin gold $(\mathrm{Au})$ layer were electron-beam evaporated without breaking the vacuum in the deposition chamber to minimize any oxidation effects. Subsequently, the nanocomposite films were spincoated onto the wafer analogously to the previous procedure. On the polymer, a $200 \mathrm{~nm}$ thin Au layer is again electron-beam evaporated and patterned by a wet-chemical etching process to form top electrodes. For our investigations, it is necessary to know the permittivity of the individual components of the composite. We measured the permittivity $\varepsilon_{r}^{m}=15$ of the $\mathrm{P}\left(\mathrm{VDF}_{70}-\mathrm{TrFE}_{30}\right)$ polymer using the LCR meter IM3533-01 (Hioki). According to the study of Wada et al., spherical $\mathrm{BaTiO}_{3}$ nanoparticles with a diameter of $120 \mathrm{~nm}$ have a permittivity of around $\varepsilon_{r}^{p} \approx 6000 .^{28}$

\section{Results and discussion}

\subsection{Randomly distributed nanocomposites and their statistics}

The upper panel in Figure 3 shows SEM images in top view of nanocomposite films with particle volume fractions of $20 \%$ and $40 \%$. In comparison, the simulated results for the total thickness function $t(x, y)$ are juxtaposed. The distributions of the nanoparticles inside of the polymer match well with the fabricated samples, i.e. the assumption of a random distribution of the particles is justified up to a volume fraction of about $40 \%$. In addition, the distribution $n\left(t_{i}\right)$ of the given total thickness function is shown. For a better overview, those pillars containing no particle material are omitted. The distribution $n\left(t_{i}\right)$ converges to a gaussian distribution as the number of particles increases. This is due to the fact that the overlaps of particle thickness functions increase with the number of particles. Otherwise, the linear distribution of a single thickness function $t^{p}(x, y)$ for spheres is always present at $v_{f} \approx 40 \%$ it is still observable, even if it is already strongly skewed. The polymer matrix in which the particles are embedded is clearly visible in the SEM image of the nanocomposite with $v_{f} \approx 20 \%$ compared to the one with $v_{f} \approx 40 \%$. Already under the latter conditions, the onset of particle agglomeration at the free upper surface is detectable due to the limited space in the matrix. 

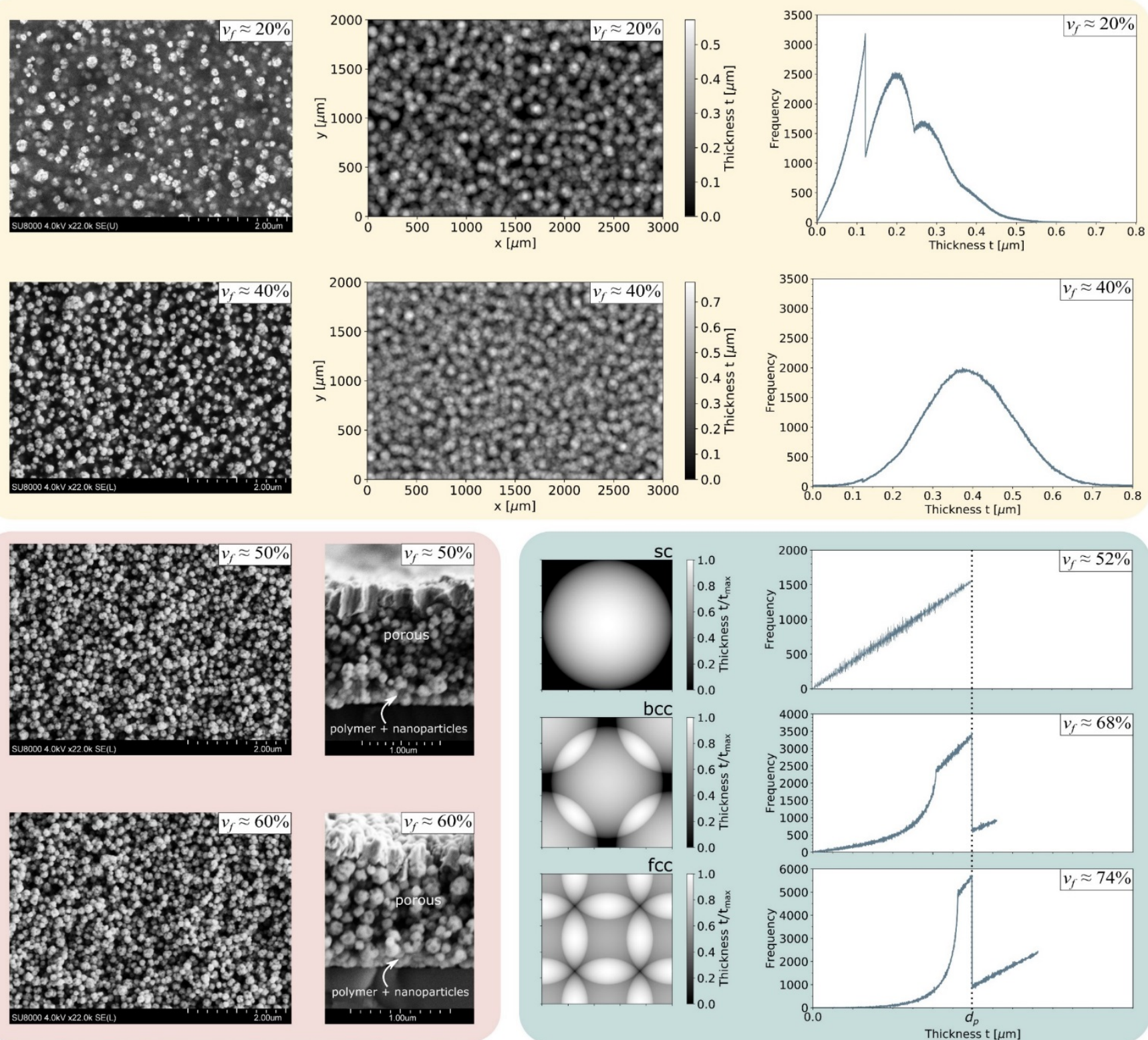

Figure 3. Upper panel: SEM images of the top view of the nanocomposite films with volume fractions of $20 \%$ and $40 \%$. In comparison, the total thickness function $t(x, y)$ as well as the corresponding distribution $n\left(t_{i}\right)$ are shown for these volume fractions. Lower left panel: SEM images in top and in cross-sectional view of the nanocomposites with volume fractions of $50 \%$ and $60 \%$. Lower right panel: Total thickness function $t(x, y)$ and the corresponding distribution $n\left(t_{i}\right)$ for basic unit cell configurations (i.e. sc, bcc and fcc) of colloidal crystals.

\subsection{Porous layer of particles and colloidal crystal formations in nanocomposites}

This is especially apparent for higher volume fractions, such as $50 \%$ or $60 \%$. Here, the particles deposit

so strongly surface-near that almost no polymer is found in the SEM images (see lower left panel of

Figure 3). In this case, we additionally analyzed the cross-sections of the nanocomposite films with the 
SEM. It turns out that a film consisting of both polymer and nanoparticles is detectable close to the silicon substrate, whereas a porous layer of particles has formed towards the top surface of the sample. Consequently, there is a characteristic value which is between a volume fraction of $40 \%$ and $50 \%$, where this effect starts to dominate. By applying a pure spin-coating process, it is not possible to achieve homogenous nanocomposite films with a higher volume fraction of spherical particles, what is confirmed by simulations of the selected particle distribution model predicting this threshold at $v_{f} \approx 40 \%$. To achieve even higher volume fractions, the spherical particles have to form a symmetric arrangement, i.e. the nanoparticles have to form colloidal crystals within the polymer matrix to reach a volume fraction of more than $50 \%$, such as a cubic (sc), body-centered cubic (bcc) or a face-centered cubic (fcc) configuration. ${ }^{25,26}$ The lower right panel of Figure 3 shows the total thickness function $t(x, y)$ and the corresponding distribution $n\left(t_{i}\right)$ for basic unit cell configurations (i.e. sc, bcc and fcc) of colloidal crystals. The symmetrical arrangement of nanoparticles as a colloidal crystal in a polymer matrix can only be achieved by more sophisticated fabrication procedures than spin-coating. ${ }^{25,26}$ When exceeding, however, a volume fraction of more than $40 \%$ in nanocomposite films realized with the latter deposition technique, these films do not show a dense and homogeneous microstructure, as particles start to accumulate close to the free surface outside of the polymer matrix, as shown in the lower left panel of Figure 3 . Furthermore, it is worth mentioning that nanocomposites of spherical particles cannot reach a volume fraction of more than $74 \%$, since this is the densest close-packing of equal spheres, although this is stated in several publications. ${ }^{14,20}$

\subsection{Permittivity of simulated and fabricated nanocomposites}

From the data presented in Figure 3, the permittivity of the nanocomposite films is calculated using equation (5). The results are shown in Figure 4. Up to a volume fraction of $40 \%$, the permittivity $\varepsilon_{r}$ of the 


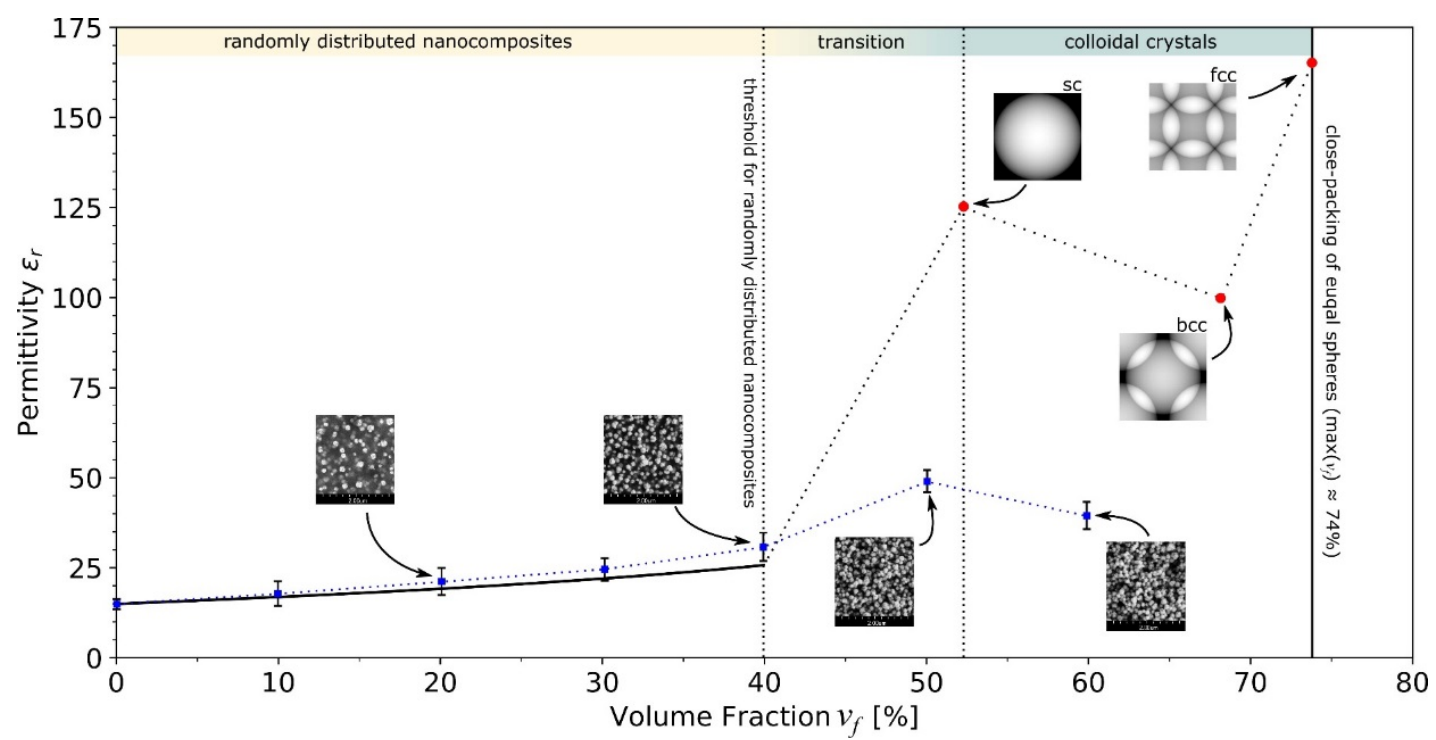

Figure 4. Simulated (-) and measured (-) permittivity values as well as those calculated for the colloidal crystal arrangement sc, bcc and fcc of spherical nanoparticles in a polymer matrix (•).

composite increases only slightly. In this regime, the calculated permittivity changes almost linearly as a function of the volume fraction with a value of only $\varepsilon_{r} \approx 24$ at $v_{f} \approx 40 \%$ and are in good agreement with the measured results. Underestimation of the experimental data are due to the fact that the particles have not all the same size and they are not perfect spheres in reality, as represented in the simulations. Above $v_{f} \approx 40 \%$, there is a transition regime in which the arrangement of the particles starts to form colloidal crystals enveloped by the polymer matrix. For the sc configuration, which is the lowest packing density of equal spheres $\left(v_{f} \approx 52 \%\right)$, the permittivity gets relatively high with a value of $\varepsilon_{r} \approx 125$ compared to the permittivity of the matrix with $\varepsilon_{r}^{m}=15$. The fcc configuration would offer a permittivity of about $\varepsilon_{r} \approx 165$, while in bcc configuration the permittivity would decrease slightly compared to sc and fcc to $\varepsilon_{r} \approx 100$.

\subsection{Analysis of variance}

As the nanoparticles are randomly distributed into the polymer matrix, the influence of this positioning procedure on the permittivity of the composite is investigated in this chapter. For this purpose, we calculate 
(a)
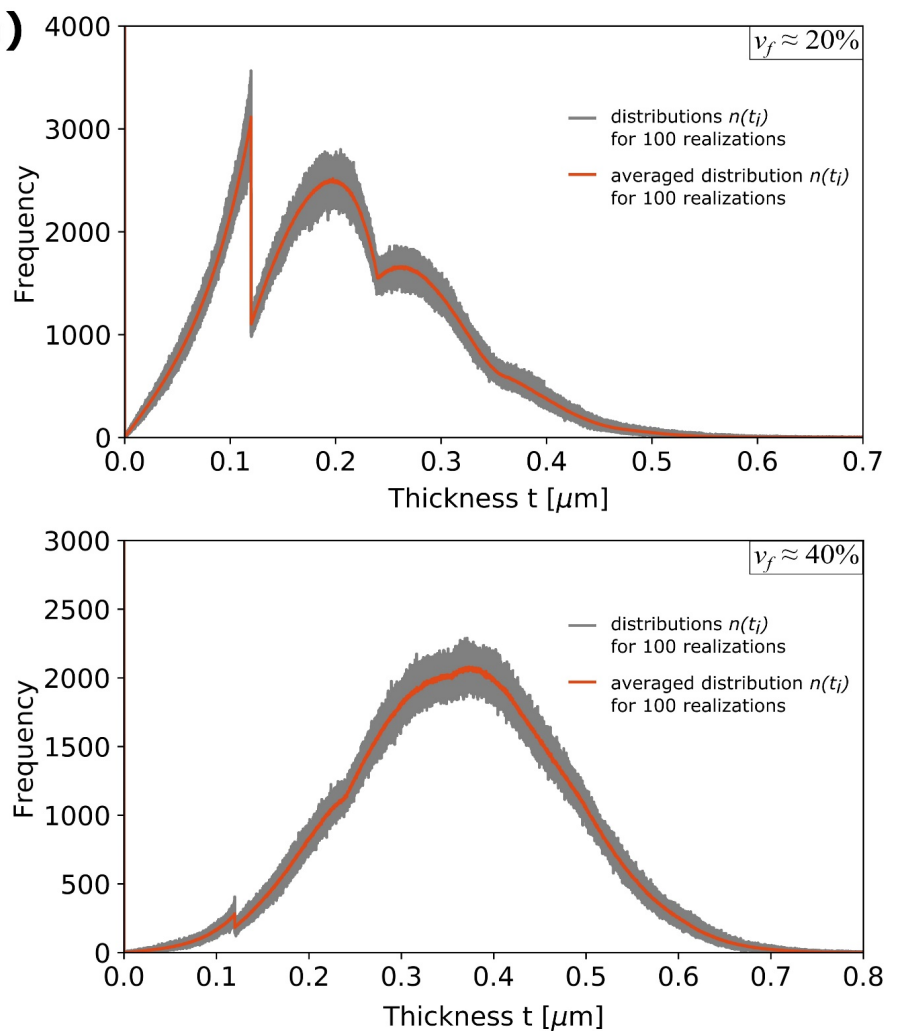

(b)
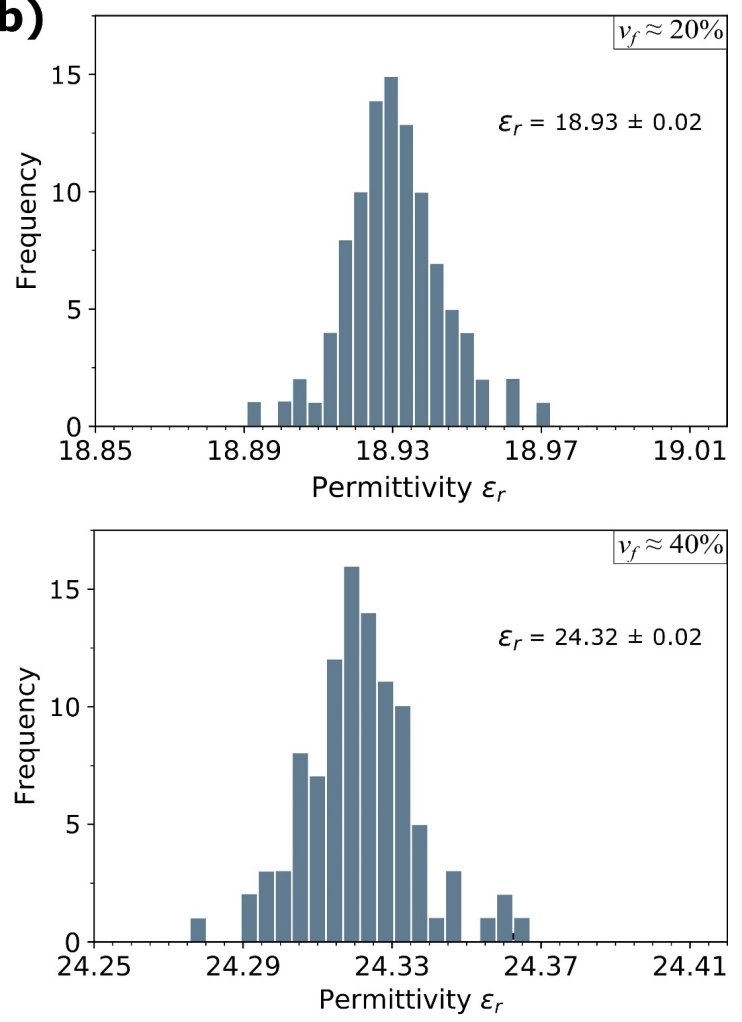

Figure 5. (a) Distribution characteristics $n\left(t_{i}\right)$ for 100 potential realization scenarios of uniformly distributed nanoparticles in a polymer matrix for a volume fraction of $20 \%$ (upper panel) und $40 \%$ (lower panel). (b) Statistics for the calculated permittivity values of the 100 different realization scenarios of (a).

calculate the permittivity values of 100 potential realization scenarios of randomly distributed polymermatrix nanocomposites. Figure 5 (a) shows 100 potential distribution characteristics $n\left(t_{i}\right)$ for volume fractions of 20 and $40 \%$, respectively. In addition, the average distribution characteristics is shown. Based on these data, a statistical analysis on the theoretically predicted permittivity of the composite is performed. Figure 5 (b) shows the histograms of the calculated permittivity values from the corresponding distributions given in Figure 5 (a). For a volume fraction of $20 \%$ the permittivity is $\varepsilon_{r}=18.93 \pm 0.02$ and for a volume fraction of $40 \%$ the permittivity is $\varepsilon_{r}=24.32 \pm 0.02$. This result indicates that realization scenarios of composites with different particle distribution of the same volume fraction should yield almost the same permittivity, resulting in a small standard deviation of only 0.02 and a variance of about 0.004 . 


\section{Conclusion}

We have introduced a novel model that allows to consider any given random distribution of nanoparticles in a polymer matrix to calculate the permittivity of dielectric nanocomposite films. Furthermore, any arbitrary particles shape can be implemented. For verification purposes, we fabricated nanocomposite films with spherical $\mathrm{BaTiO}_{3}$ nanoparticles admixed into a $\mathrm{P}\left(\mathrm{VDF}_{70}-\mathrm{TrFE}_{30}\right)$ polymer and compared our theoretically predicted results with measurement data. We showed that spin-coated nanocomposites with a volume fraction up to $v_{f} \approx 40 \%$ result in homogenous films without any porous layer forming close to the free surface. For higher particle volume fractions, however, more complex fabrication processes are required, to support the transition to colloidal crystal formation. In addition, we were able to predict theoretically the permittivity of nanocomposites containing colloidal crystals for the first time. This is of utmost importance for future device applications requiring high permittivity materials, as this material parameter increases by more than an order of magnitude compared to the pure polymer matrix. Due to this theoretical basis, dielectric properties of complex nanocomposite materials are estimated and tailored to their physical limits. For future investigations, the model presented in this study need to extended. To increase its accuracy, the presence of an interphase between nanoparticles and polymer matrix ${ }^{29-31}$, the size distribution of the particles or agglomeration effects should be integrated. Furthermore, a random close packing code ${ }^{32,33}$ could arrange the particles (spheres) more tightly and thus it would be possible to resolve higher volume fractions in more detail.

\section{Acknowledgement}

This work has received funding from the European Union`s Horizon 2020 research and Innovation programme under the Marie Sklodowska-Curie grant agreement No. 721874 (SPM2.0). 


\section{References}

1. MacChesney, J. B.; Gallagher, P. K.; DiMarcello, F. V. Stabilized Barium Titanate Ceramics for Capacitor Dielectrics. Journal of the American Ceramic Society 1963, 46 (5), 197-202.

2. Li, W. B.; Zhou, D.; Pang, L. X.; Xu, R.; Guo, H. H. Novel barium titanate based capacitors with high energy density and fast discharge performance. Journal of Materials Chemistry A 2017, 37.

3. Kingon, A. I.; Srinivasan, S. Lead zirconate titanate thin films directly on copper electrodes for ferroelectric, dielectric and piezoelectric applications. Nature Materials 2005, 4, 233-237.

4. Singh, M.; Yadav, B. C.; Ranjan, A.; Kaur, M.; Gupta, S. K. Synthesis and characterization of perovskite barium titanate thin film and its application as LPG sensor. Sensors and Actuators B: Chemical 2017, 241, 1170-1178.

5. Sikarwar, S.; Sonker, R. K.; Shukla, A.; Yadav, B. C. Synthesis and investigation of cubical shaped barium titanate and its application as opto-electronic humidity sensor. Journal of Materials Science: Materials in Electronics 2018, 29 (15), 12951-12958.

6. Zhu, J.; Ren, L.; Ho, S. C.; Jia, Z.; Song, G. Gas pipeline leakage detection based on PZT sensors. Smart Materials and Structures 2017, 26 (2).

7. Burcsu, E.; Ravichandran, G.; Bhattacharya, K. Large strain electrostrictive actuation in barium titanate. Applied Physics Letters 2000, 77, 1968.

8. Harris, N. R.; Hill, M.; Torah, R.; Townsend, R.; Beeby, S.; White, N. M.; Ding, J. A multilayer thick-film PZT actuator for MEMS applications. Sensors and Actuators A: Physical 2006, 132 (1), 311-316.

9. Roberts, S. Dielectric and Piezoelectric Properties of Barium Titanate. Physical Review 1947, 71, 890.

10. Jaffe, B.; Roth, R. S.; Marzullo, S. Piezoelectric Properties of Lead Zirconate-Lead Titanate SolidSolution Ceramics. Journal of Applied Physics 1954, 25, 809. 
11. Tunkasiri, T.; Rujijanagul, G. Dielectric strength of fine grained barium titanate ceramics. Journal of Materials Science Letters 1996, 15, 1767-1769.

12. Udayakumar, K. R.; Schuele, P. J.; Chen, J.; Krupanidhi, S. B.; Cross, L. E. Thickness-dependent electrical characteristics of lead zirconate titanate thin films. Journal of Applied Physics 1995, 77, 3981.

13. Jow, T. R.; Cygan, P. J. Dielectric breakdown of polyvinylidene fluoride and its comparisons with other polymers. Journal of Applied Physics 1993, 73, 5147.

14. Kim, P.; Doss, N. M.; Tillotson, J. P.; Hotchkiss, P. J.; Pan, M. J.; Marder, S. R.; Li, J.; Calame, J. P.; Perry, J. W. High Energy Density Nanocomposites Based on Surface-Modified $\mathrm{BaTiO}_{3}$ and a Ferroelectric Polymer. ACS Nano 2009, 3 (9), 2581-2592.

15. Dou, X.; Liu, X.; Zhang, Y.; Feng, H.; Chen, J. F.; Du, S. Improved dielectric strength of barium titanate-polyvinylidene fluoride nanocomposites. Applied Physics Letters 2009, 95, 132904.

16. Wang, Y.; Cui, J.; Yuan, Q.; Niu, Y.; Bai, Y.; Wang, H. Significantly Enhanced Breakdown Strength and Energy Density in Sandwich-Structured Barium Titanate/ Poly(vinylidene fluoride) Nanocomposites. Advanced Materials 2015, 27 (42), 6658-6663.

17. Chan, H. L. W.; Cheung, M. C.; Choy, C. L. Study on $\mathrm{BaTiO}_{3} / \mathrm{P}(\mathrm{VDF}-\mathrm{TrFE})$ 0-3 composites. Ferroelectrics 1999, 224 (1), 113-120.

18. Li, Q.; Chen, L.; Gadinski, M. R.; Zhang, S.; Zhang, G.; Li, H. U.; Iagodkine, E.; Haque, A.; Chen, L. Q.; Jackson, T. N.; Wang, Q. Flexible high-temperature dielectric materials from polymer nanocomposites. Nature 2015, 523, 576-579.

19. Moussa, M.; El-Kady, M. F.; Abdel-Azeim, S.; Kaner, R. B.; Majewski, P.; Ma, J. Compact, flexible conducting polymer/graphene nanocomposites for supercapacitors of high volumetric energy density. Composites Science and Technology 2018, 160, 50-59. 
20. Ezzat, M.; Sabhia, N. A.; Izzularab, M. Accurate model for computing dielectric constant of dielectric nanocomposites. Applied Nanoscience 2014, 4, 331-338.

21. Hamdia, K. M.; Silani, M.; Zhuang, X.; He, P.; Rabczuk, T. Stochastic analysis of the fracture thoughness of polymeric nanoparticle composites using polynomial chaos expansions. International Journal of Fracture 2017, 206, 215-227.

22. Msekh, M. A.; Cuong, N. H.; Zi, G.; Areia, P.; Zhuang, X.; Rabczuk, T. Fracture properties prediction of clay/epoxy nanocomposites with interphase zones using a phase field model. Engineering Fracture Mechanics 2018, 188, 287-299.

23. Yamada, T. T. Piezoelectricity of hight-content lead zirconate titanate/polymer composite. Journal of Applied Physics 1982, 53, 4328.

24. Goncharenko, A. V.; Lozovski, V. Z.; Venger, E. F. Lichtenecker's equation: applicability and limitations. Optics Communications 2000, 174, 19-32.

25. Cong, H.; Yu, B.; Tang, J.; Li, Z.; Liu, X. Current status and future developments in preparation and application of colloidal crystals. Chemical Society Review 2013, 42, 7774.

26. Fang, Y.; Phillips, B. M.; Askar, K.; Choi, B.; Jiang, P.; Jiang, B. Scalable bottom-up fabrication of colloidal photonic crystals and periodic plasmonic nanostructures. Journal of Materials Chemistry 2013, 1, 6031.

27. Vu-Bac, N.; Rafiee, R.; Zhuang, X.; Lahmer, T.; Rabczuk, T. Uncertainty quantification for multiscale modeling of polymer nanocomposites with correlated parameters. Composites Part B 2015, 68, 446-464.

28. Wada, S.; Yasuno, H.; Hoshina, T.; Nam, S. N.; Kakemoto, H.; Tsurumi, T. Preparation of nmsized barium titanate fine particles and their powder. Japanese Journal of Applied Physics 2003, $42(1), 9 B$. 
29. Zammarano, M.; Maupin, P. H.; Sung, L. P.; Gilman, J. W.; McCarthy, E. D.; Kim, Y. S.; Fox, D. M. Revealing the Interphase in Polymer Nanocomposites. ACS Nano 2011, 5 (4), 3391-3399.

30. Li, J. Y.; Huang, C.; Zhang, Q. Enhanced Electromechanical Properties in All-Polymer Percolative Composites. Applied Physics Letters 2004, 84, 3124-3126.

31. Qiao, R.; Brinson, L. Simulation of interphase percolation and gradients in polymer nanocomposites. Composites Science and Technology 2008, 69, 491-499.

32. Scott, G. D.; Kilgour, D. M. The density of random close packing of spheres. Journal of Physics D: Applied Physics 1969, 2, 863.

33. Berryman, J. G. Random close packing of hard spheres and disks. Physical Review A 1983, 27, 1053. 